\title{
Parameter Estimations for Some Modifications of the Weibull Distribution
}

\author{
Soumaya Ghnimi ${ }^{1}$, Soufiane Gasmi ${ }^{2}$ \\ ${ }^{1}$ Faculty of Sciences of Tunis, University of Tunis El Manar, Tunis, Tunisia \\ ${ }^{2}$ Tunis National Higher School of Engineering, University of Tunis, Tunis, Tunisia \\ Email: soufiane.kasmi3@gmail.com
}

Received 20 May 2014; revised 26 June 2014; accepted 12 July 2014

Copyright (C) 2014 by authors and Scientific Research Publishing Inc.

This work is licensed under the Creative Commons Attribution International License (CC BY). http://creativecommons.org/licenses/by/4.0/

c) (i) Open Access

\begin{abstract}
Proposed by the Swedish engineer and mathematician Ernst Hjalmar Waloddi Weibull (18871979), the Weibull distribution is a probability distribution that is widely used to model lifetime data. Because of its flexibility, some modifications of the Weibull distribution have been made from several researches in order to best adjust the non-monotonic shapes. This paper gives a study on the performance of two specific modifications of the Weibull distribution which are the exponentiated Weibull distribution and the additive Weibull distribution.
\end{abstract}

\section{Keywords}

Exponentiated Weibull Distribution, Additive Weibull Distribution, Maximum Likelihood Estimation, Kolmogorov-Smirnov Test, Simulation

\section{Introduction}

The Weibull distribution [1] is the most life-time probability distribution used in the reliability engineering discipline. Due to its wide applications [2], many researchers have developed various extensions and modified forms of the Weibull distribution with a number of parameters ranging from 2 to 5 . These distributions have several desirable properties and nice physical interpretations. The literature that studies the various modifications of the Weibull distributions is extensive, for example: the two-parameter flexible Weibull extension of Bebbington et al. [3]. Zhang and Xie [4] studied the characteristics and application of the truncated Weibull distribution which has a bathtub shaped hazard function.

A three-parameter model, called exponentiated Weibull distribution, was introduced by Mudholkar and Sri- 
vastave [5]. The modified Weibull distribution of Sarhan and Zaindin [6] was studied by Gasmi and Berzig [7] in the case of type I censored data. Another three-parameter model was developed by Marshall and Olkin [8] and is called the extended Weibull distribution. Xie et al. [9] proposed a three-parameter modified Weibull extension with a bathtub shaped hazard function. Lai et al. [10] have described the modified Weibull (MW) distribution. A four-parameter additive Weibull distribution (AddW) was proposed by Xie and Lai [11]. A second four-parameter beta Weibull distribution was proposed by Famoye et al. [12]. Cordeiro et al. [13] introduced another four-parameter distribution called the Kumaraswamy Weibull distribution. A five-parameter modified Weibull distribution was introduced by Phani [14]. The beta modified Weibull distribution was introduced by Silva et al. [15] and further studied by Cordeiro et al. [16]. Recently, an extensive review of some discrete and continuous versions of the modifications of the Weibull distribution was introduced by Almalki and Nadarajah [17]. The main objective of this article is in first step to estimate the three unknown parameters of the exponentiated Weibull distribution and the four unknown parameters of the additive Weibull distribution. Therefore, we use the maximum likelihood method to derive such estimates. In the second step, we study whether these distributions fit a set of real data of Aarset [18] better than other distributions. Two criteria are used for this purpose: the first one is the mean square distance MSD and the next one is the Kolmogorov-Smirnov test statistic. A real data set is analyzed and it is observed that the present distributions provide better fit than many existing well-known distributions. This paper will be organized as follows. In Section 2 we present the exponentiated Weibull distribution and the additive Weibull distribution. In Section 3, an application to real data is provided and different types of goodness-of-fit are applied to test the compatibility of the exponentiated Weibull distribution and the additive Weibull distribution in comparison to some other models. Mainly we use the mean square distance MSD and the Kolmogorov-Smirnov (K-S) test as a non-parametric test to illustrate how one can compare the exponentiated Weibull distribution and the additive Weibull distribution with some sub-models. Finally we conclude the paper in Section 4.

\section{Parameter Estimates of EW and AddW Distributions}

\subsection{Exponentiated Weibull Distribution}

The exponentiated Weibull (EW) distribution is proposed by Mudholkar and Srivastava [5] and studied first by Mudholkar et al. [19] and further by Mudholkar and Hutson [20].

The cumulative distribution function (CDF) and the survival function of the EW distribution, denoted by $\operatorname{EW}(\alpha, \theta, \lambda)$ are respectively:

$$
F_{\mathrm{EW}}(t ; \Theta)=\left(1-\exp \left(-\alpha t^{\theta}\right)\right)^{\lambda}, \text { where } \Theta=(\alpha, \theta, \lambda) \text { and } \alpha, \theta, \lambda>0
$$

and

$$
S_{\mathrm{EW}}(t ; \Theta)=1-\left(1-\exp \left(-\alpha t^{\theta}\right)\right)^{\lambda}
$$

The $\operatorname{EW}(\alpha, \theta, \lambda)$ distribution generalizes the following distributions: 1$)$ exponential distribution $\operatorname{ED}(\alpha)$ by setting $\theta=1, \lambda=1,2)$ Rayleigh distribution $\operatorname{RD}(\alpha)$ by setting $\theta=2, \lambda=1,3$ ) generalized exponential distribution $\operatorname{GED}(\alpha, \lambda)$ [21] by setting $\theta=1$ and 4) Weibull distribution $\operatorname{WD}(\alpha, \theta)$ [22] [23] by setting $\lambda=1$.

Figure 1 represents the cumulative distribution function and the survival function of the $\operatorname{EW}(\alpha, \theta, \lambda)$ for different values of $\alpha, \theta$ and $\lambda$.

The probability density function of the $\operatorname{EW}(\alpha, \theta, \lambda)$ distribution is given by:

$$
f(t ; \Theta)=\lambda \alpha \theta t^{\theta-1} \exp \left(-\alpha t^{\theta}\right)\left(1-\exp \left(-\alpha t^{\theta}\right)\right)^{\lambda-1}, t>0 .
$$

The corresponding hazard function has the form:

$$
h(t ; \Theta)=\frac{\lambda \alpha \theta t^{\theta-1} \exp \left(-\alpha t^{\theta}\right)\left(1-\exp \left(-\alpha t^{\theta}\right)\right)^{\lambda-1}}{1-\left(1-\exp \left(-\alpha t^{\theta}\right)\right)} .
$$

Figure 2 shows respectively the probability density function and the hazard rate function of the $\operatorname{EW}(\alpha, \theta, \lambda)$ 

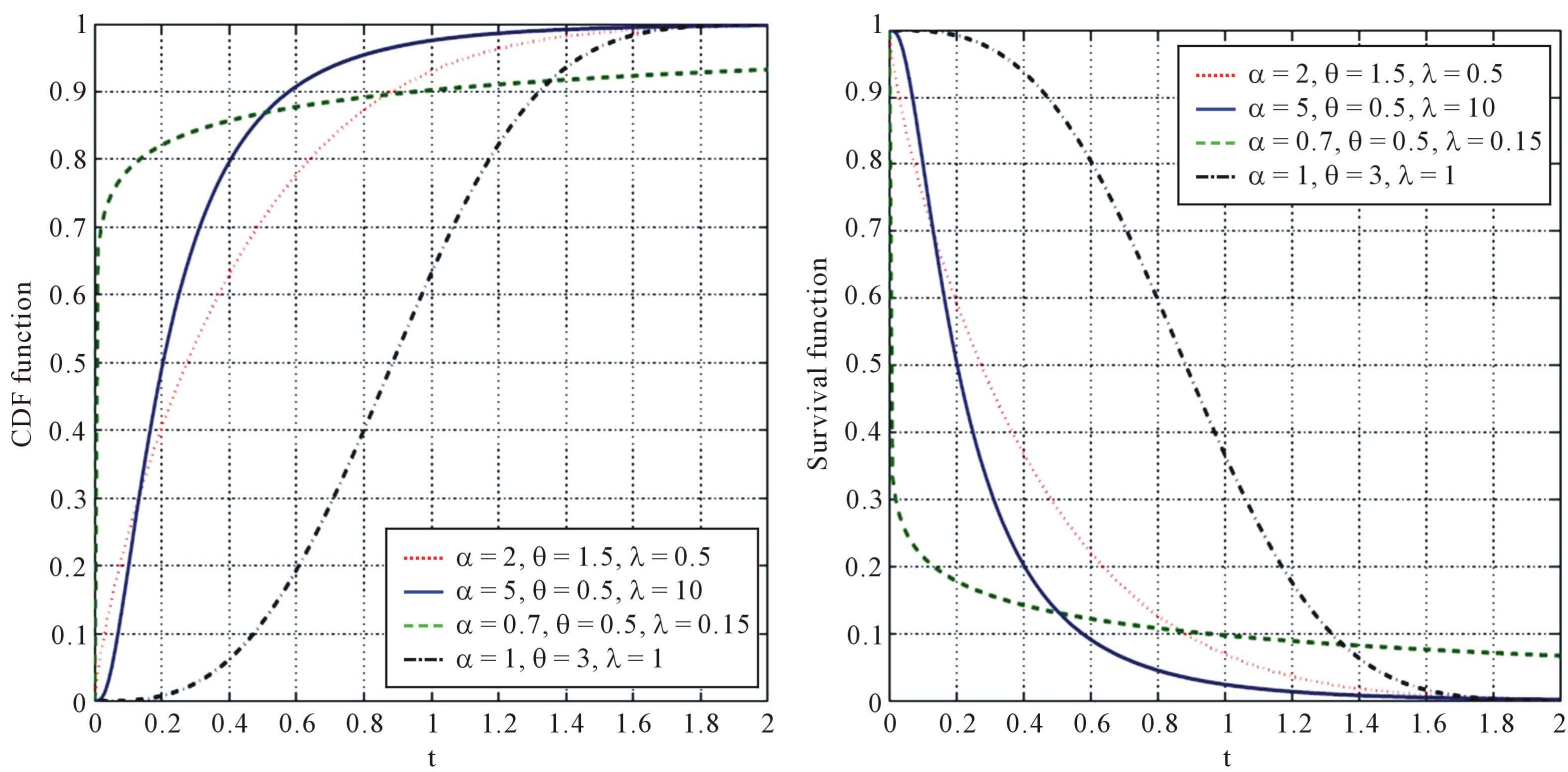

Figure 1. Plots of cumulative distribution function and survival function of $\operatorname{EW}(\alpha, \theta, \lambda)$.
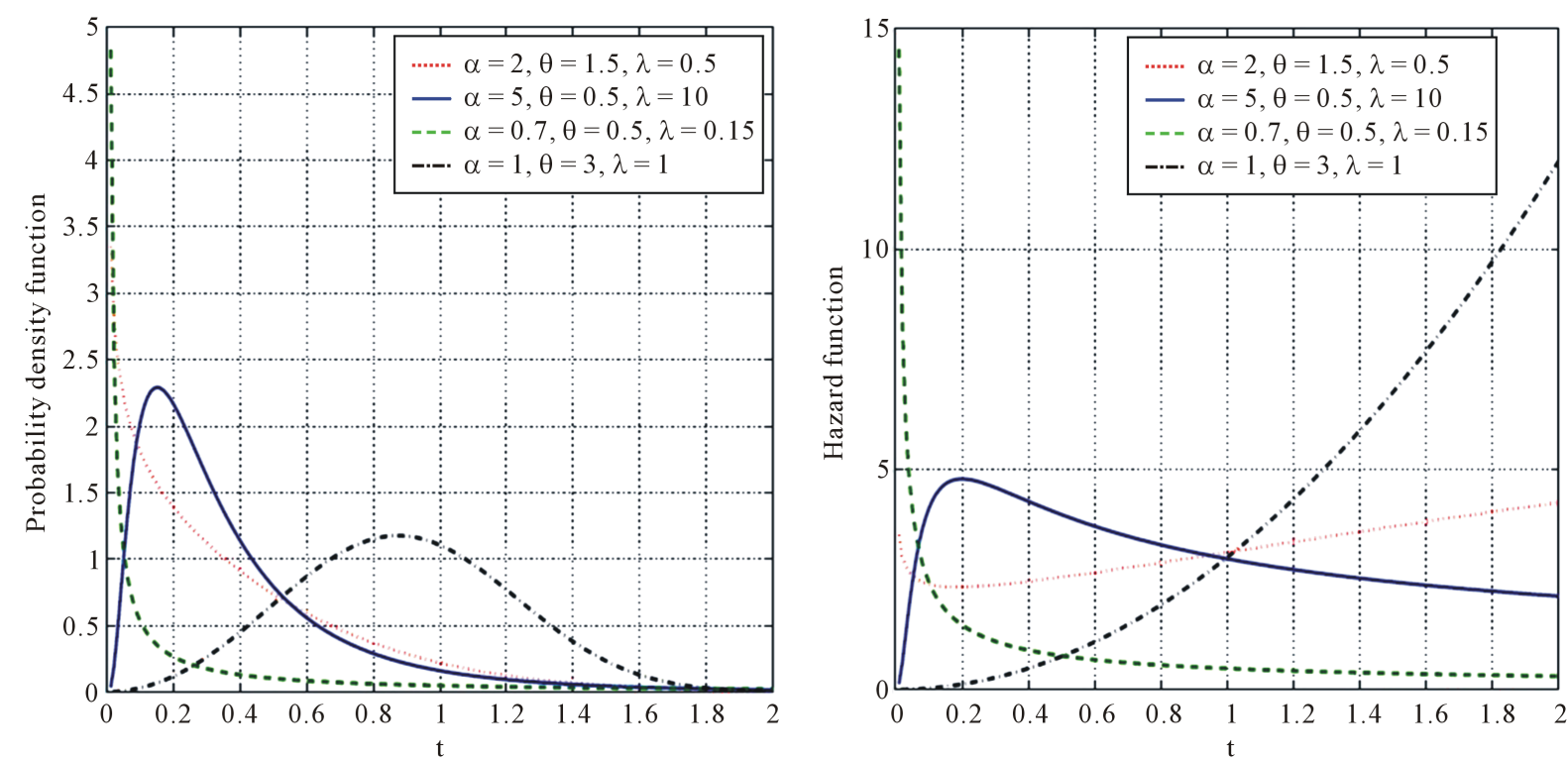

Figure 2. Plots of probability density function and hazard rate function of the $\operatorname{EW}(\alpha, \theta, \lambda)$.

distribution for different values of $\alpha, \theta$ and $\lambda$.

\subsubsection{Data Simulations of the $\operatorname{EW}(\alpha, \theta, \lambda)$ Distribution}

By setting the three parameters $\alpha, \theta$ and $\lambda$ as follows: $\alpha=1, \theta=3$ and $\lambda=1$, we obtain simulation data of a $\operatorname{EW}(\alpha, \theta, \lambda)$ distribution. We remark that the $\operatorname{EW}(\alpha, \theta, \lambda)$ distribution has the advantage that it possessed a closed form of cdf, therefore we can generate random values from it by using the explicit formula:

$$
t_{i}=\left(\frac{-\log \left(1-U^{1 / \lambda}\right)}{\alpha}\right)^{1 / \theta},
$$

where $i=1, \cdots, n, n$ is the sample size and $U$ is a uniformly distributed random variable on the interval $(0,1)$. 
Figure 3 illustrate the empirical cdf, the cdf and the 95\% lower and upper confidence bounds for the cdf of 100 simulated data by setting $\alpha=1, \theta=3$ and $\lambda=1$.

\subsubsection{Parameter Estimation}

To estimate the parameters of the $\operatorname{EW}(\alpha, \theta, \lambda)$ distribution we use the maximum-likelihood method which is a traditional parametric method to estimate the parameters and has good properties such as asymptotic normality and consistency. Suppose now that we have a random sample, $\left(t_{1}, t_{2}, \cdots, t_{n}\right)$ of a $\operatorname{EW}(\alpha, \theta, \lambda)$ distribution with unknown parameter vector $\Theta=(\alpha, \theta, \lambda)$. The likelihood function of $\Theta$ is given by:

$$
L\left(t_{i} ; \Theta\right)=\prod_{i=1}^{n} \lambda \alpha \theta t_{i}^{\theta-1} \exp \left(-\alpha t_{i}^{\theta}\right)\left(1-\exp \left(-\alpha t_{i}^{\theta}\right)\right)^{\lambda-1} .
$$

The log-likelihood function has the following form:

$$
L\left(t_{1} ; \Theta\right)=\prod_{i=1}^{n} \lambda \alpha \theta t_{i}^{\theta-1} \exp \left(-\alpha t_{1}^{\theta}\right)\left(1-\exp \left(-\alpha t_{1}^{\theta}\right)\right)^{\lambda-1} .
$$

After calculating the first partial derivatives of $\ln L\left(t_{1} ; \Theta\right)$ and setting the results to zero, we get the following score functions:

$$
\begin{gathered}
0=\frac{n}{\alpha}-\sum_{i=1}^{n} t_{i}^{\theta}+(\lambda-1) \sum_{i=1}^{n} \frac{t_{i}^{\theta} \mathrm{e}^{-\alpha t_{i}^{\theta}}}{1-\mathrm{e}^{-\alpha t_{i}^{\theta}}} \\
0=\frac{n}{\theta}+\sum_{i=1}^{n} \log \left(t_{i}\right)-\alpha \sum_{i=1}^{n} \log \left(t_{i}\right) t_{i}^{\theta}+\alpha(\lambda-1) \sum_{i=1}^{n} \frac{\log \left(t_{i}\right) t_{i}^{\theta} \mathrm{e}^{-\alpha t_{i}^{\theta}}}{1-\mathrm{e}^{-\alpha t_{i}^{\theta}}} \\
0=\frac{n}{\lambda}+\sum_{i=1}^{n} \log \left(1-\mathrm{e}^{-\alpha t_{i}^{\theta}}\right)
\end{gathered}
$$

To get the MLE of the parameters $\alpha, \theta$ and $\lambda$ we have to solve the above system of three non-linear equations with respect to $\alpha, \theta$ and $\lambda$. The solution of this system of equations is not possible in closed form, so numerical technique such as the trust region method, which requires the second derivatives of the $\ln L\left(t_{1} ; \Theta\right)$ function is needed to get the MLE. We note that in order to accelerate the resolution of the system (7), (8), (9) by using the software MATLAB, we have introduced the following second partial derivatives of $\ln L\left(t_{1} ; \Theta\right)$ :

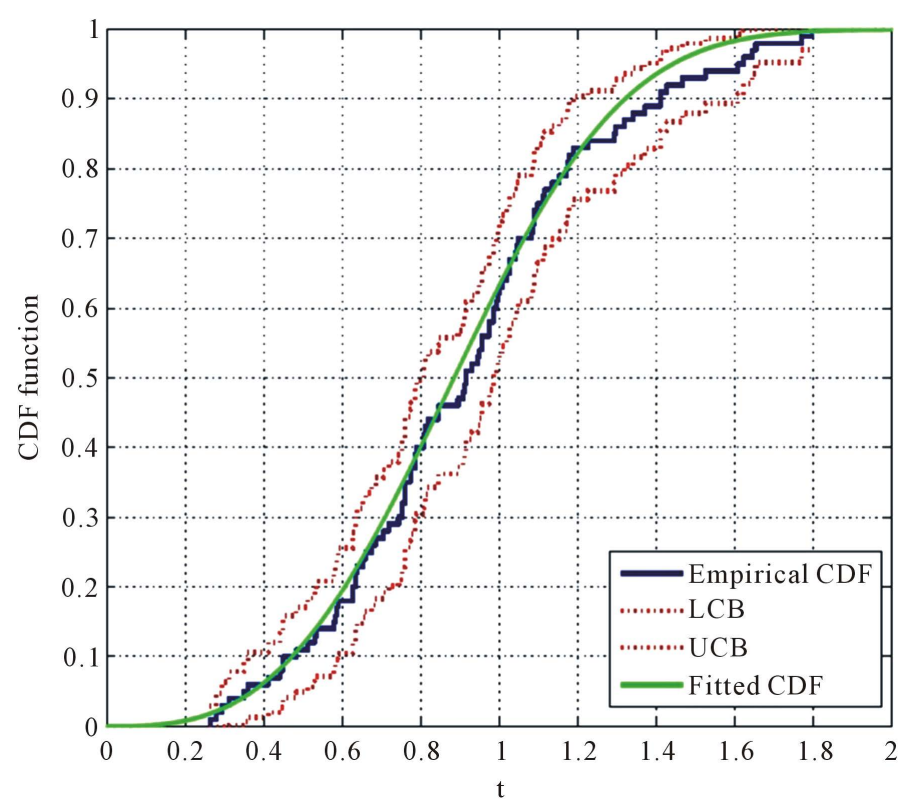

Figure 3. Cdf and empirical cdf of the $\operatorname{EW}(\alpha, \theta, \lambda)$ distribution. 


$$
\begin{gathered}
\frac{\partial^{2} \ln L}{\partial \alpha^{2}}=-\frac{n}{\alpha^{2}}-(\lambda-1) \sum_{i=1}^{n} \frac{t_{i}^{2 \theta} \mathrm{e}^{-\alpha t_{i}^{\theta}}}{\left(1-\mathrm{e}^{-\alpha t_{i}^{\theta}}\right)^{2}} \\
\frac{\partial^{2} \ln L}{\partial \theta^{2}}=-\frac{n}{\theta^{2}}-\alpha \sum_{i=1}^{n} \log ^{2}\left(t_{i}\right) t_{i}^{\theta}+\alpha(\lambda-1) \sum_{i=1}^{n} \frac{\log ^{2}\left(t_{i}\right) t_{i}^{\theta} \mathrm{e}^{-\alpha t_{i}^{\theta}}\left(1-\alpha t_{i}^{\theta}-\mathrm{e}^{-\alpha t_{i}^{\theta}}\right)}{\left(1-\mathrm{e}^{-\alpha t_{i}^{\theta}}\right)^{2}} \\
\frac{\partial^{2} \ln L}{\partial \lambda^{2}}=-\frac{n}{\lambda^{2}} \\
\frac{\partial^{2} \ln L}{\partial \alpha \partial \theta}=-\sum_{i=1}^{n} \log \left(t_{i}\right) t_{i}^{\theta}+(\lambda-1) \sum_{i=1}^{n} \frac{\log \left(t_{i}\right) t_{i}^{\theta} \mathrm{e}^{-\alpha t_{i}^{\theta}}\left(1-\alpha t_{i}^{\theta}-\mathrm{e}^{-\alpha t_{i}^{\theta}}\right)}{\left(1-\mathrm{e}^{-\alpha t_{i}^{\theta}}\right)^{2}} \\
\frac{\partial^{2} \ln L}{\partial \alpha \partial \lambda}=\sum_{i=1}^{n} \frac{t_{i}^{\theta} \mathrm{e}^{-\alpha t_{i}^{\theta}}}{1-\mathrm{e}^{-\alpha t_{i}^{\theta}}} \\
\frac{\partial^{2} \ln L}{\partial \lambda \partial \theta}=\alpha \sum_{i=1}^{n} \frac{\log \left(t_{i}\right) t_{i}^{\theta} \mathrm{e}^{-\alpha t_{i}^{\theta}}}{1-\mathrm{e}^{-\alpha t_{i}^{\theta}}} .
\end{gathered}
$$

Table 1 gives the estimated parameters of $N=10$ simulations and the mean square error of each parameter, where:

$$
\operatorname{MSE}(\hat{\Theta})=\frac{1}{N} \sum_{i=1}^{N}\left(\Theta-\hat{\Theta}_{i}\right)^{2}
$$

\subsection{Additive Weibull Distribution}

The additive Weibull (AddW) distribution has four parameters $\alpha, \beta, \theta$ and $\gamma$. This distribution is first introduced by Xie and Lai [11] and is denoted by $\operatorname{AddW}(\alpha, \beta, \theta, \gamma)$. We remark, that this distribution has a bathtub shaped hazard function and it was obtained as the sum of two hazard functions of Weibull distributions.

The cumulative distribution function of the $\operatorname{AddW}(\alpha, \beta, \theta, \gamma)$ is defined as follows:

Table 1. Parameter estimates of $\alpha=1, \theta=3$ and $\lambda=1$.

\begin{tabular}{cccc}
\hline & \multicolumn{3}{c}{ ML } \\
\cline { 2 - 3 } & $\hat{\alpha}$ & $\hat{\theta}$ & $\hat{\lambda}$ \\
\hline 1.1002 & 2.9859 & 1.0415 \\
1.0217 & 3.0602 & 1.0954 \\
1.0930 & 3.0329 & 0.9756 \\
1.0293 & 3.0174 & 1.0183 \\
& 1.0724 & 3.0286 & 0.9842 \\
& 1.0903 & 3.0802 & 0.9988 \\
& 0.9929 & 3.0472 & 0.9904 \\
MSE & 1.0081 & 2.9723 & 1.0480 \\
& 0.9905 & 2.9729 & 0.9863 \\
& 0.9897 & 3.0126 & 1.0208 \\
& 0.0026 & 0.0016 & 0.0015 \\
\hline
\end{tabular}




$$
F_{\text {Addw }}(t ; \Theta)=1-\exp \left(-\alpha t^{\theta}-\beta t^{\gamma}\right) \text {, where } \Theta=(\alpha, \beta, \theta, \gamma), \quad \alpha, \beta, \theta>0 \text { and } \gamma<1 .
$$

The corresponding survival function is:

$$
S_{\text {Addw }}(t ; \Theta)=\exp \left(-\alpha t^{\theta}-\beta t^{\gamma}\right) .
$$

The $\operatorname{AddW}(\alpha, \beta, \theta, \gamma)$ distribution generalizes the following distributions: 1$)$ linear failure rate distribution $\operatorname{LRFD}(\alpha, \beta)$ [7] by setting $\gamma=2$ and $\theta=1$,2) Weibull distribution $\operatorname{WD}(\alpha, \theta)$ by setting $\beta=0$ and 3) modified Weibull distribution $\operatorname{MWD}(\alpha, \beta, \gamma)$ [10] by setting $\theta=1$.

Figure 4 shows respectively the cumulative distribution function and the survival function of the additive Weibull distribution for different values of $\alpha, \beta, \theta$ and $\gamma$.

The probability density function of the $\operatorname{AddW}(\alpha, \beta, \theta, \gamma)$ distribution is given by:

$$
f(t ; \Theta)=\left(-\alpha t^{\theta-1}-\beta t^{\gamma-1}\right) \exp \left(-\alpha t^{\theta}-\beta t^{\gamma}\right), t>0
$$

The corresponding hazard function has the form:

$$
h(t ; \Theta)=\alpha \theta t^{\theta-1}+\beta \gamma t^{\gamma-1}
$$

Figure 5 shows the probability density function and the hazard rate function of the $\operatorname{AddW}(\alpha, \beta, \theta, \gamma)$ distribution for different values of $\alpha, \beta, \theta$ and $\gamma$.

\subsubsection{Data Simulations of the $\operatorname{AddW}(\alpha, \beta, \theta, \gamma)$ Distribution}

By setting the four parameters $\alpha, \beta, \theta$ and $\gamma$ as follows: $\alpha=1.5, \beta=0.5, \theta=3$ and $\gamma=0.8$, we obtain simulation data of the $\operatorname{AddW}(\alpha, \beta, \theta, \gamma)$ distribution. We generate random values from it by solving the following equation:

$$
\log (1-U)+\alpha t_{i}^{\theta}+\beta t_{i}^{\gamma}=0
$$

where $i=1, \cdots, n, n$ is the sample size and $U$ is a uniformly distributed random variable on the interval $(0,1)$.

Figure 6 illustrates the empirical cdf, the cdf and the 95\% lower and upper confidence bounds for the cdf of the 100 simulated data by setting $\alpha=1.5, \beta=0.5, \theta=3$ and $\gamma=0.8$.

\subsubsection{Parameter Estimation}

Now, we introduce the estimation of the model parameters by using the method of maximum likelihood. Let
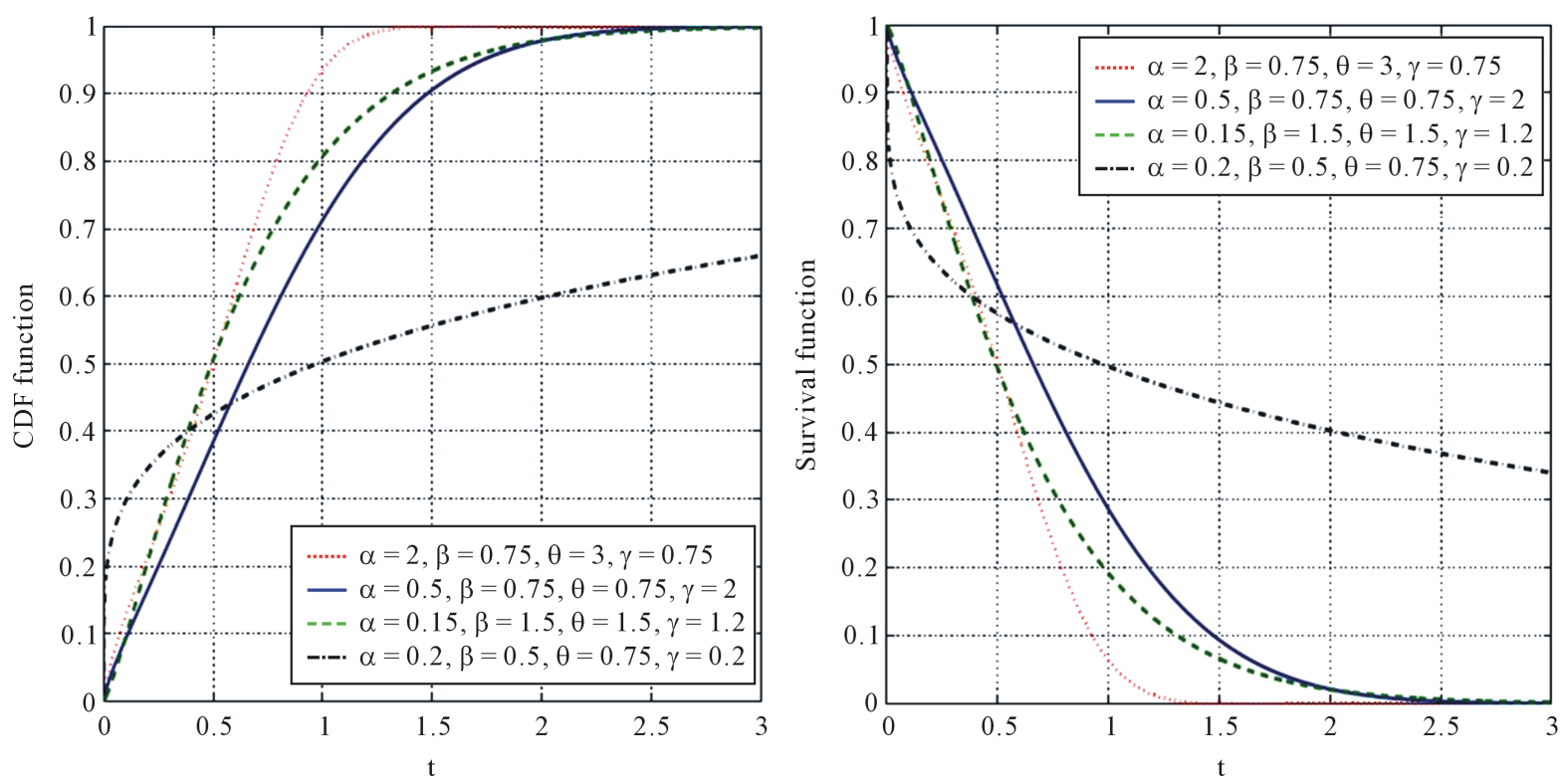

Figure 4. Plots of cumulative distribution function and survival function of the $\operatorname{AddW}(\alpha, \beta, \theta, \gamma)$. 

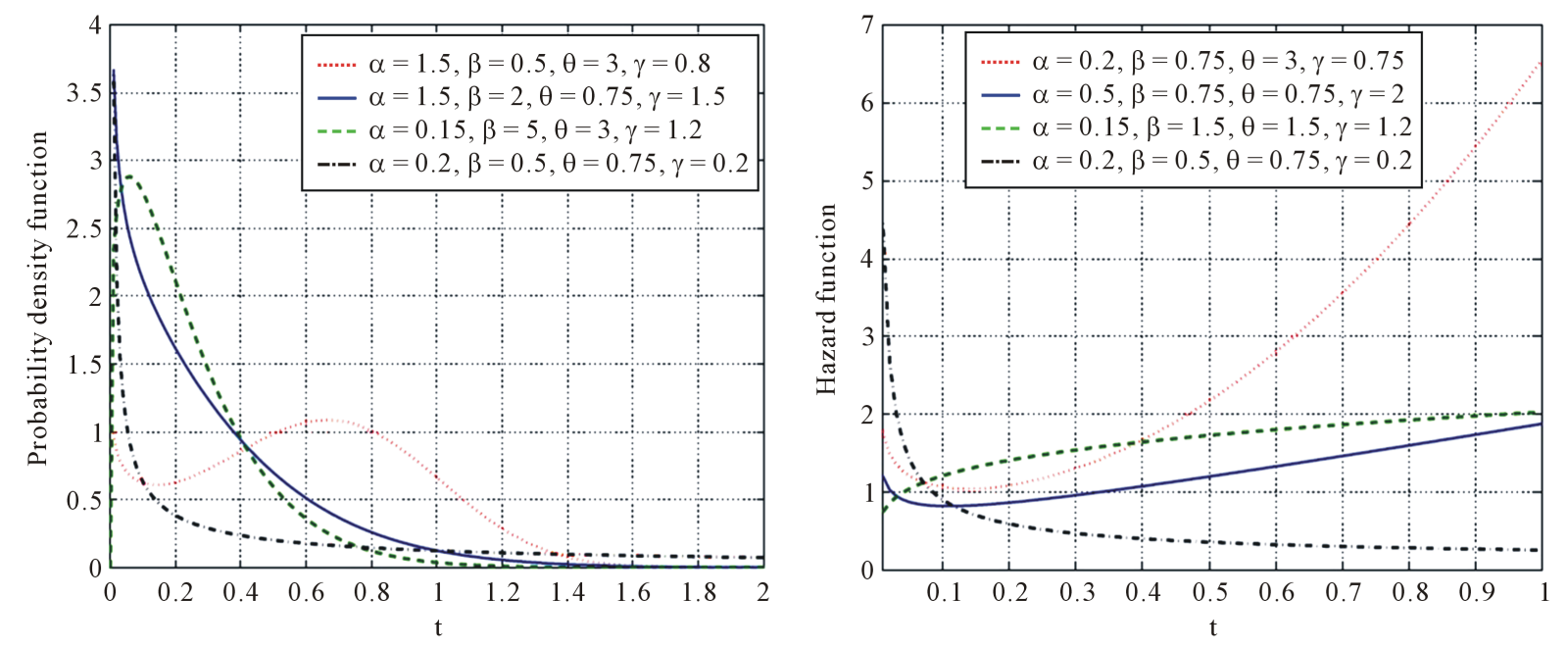

Figure 5. Plots of probability density function and hazard rate function of the $\operatorname{AddW}(\alpha, \beta, \theta, \gamma)$.

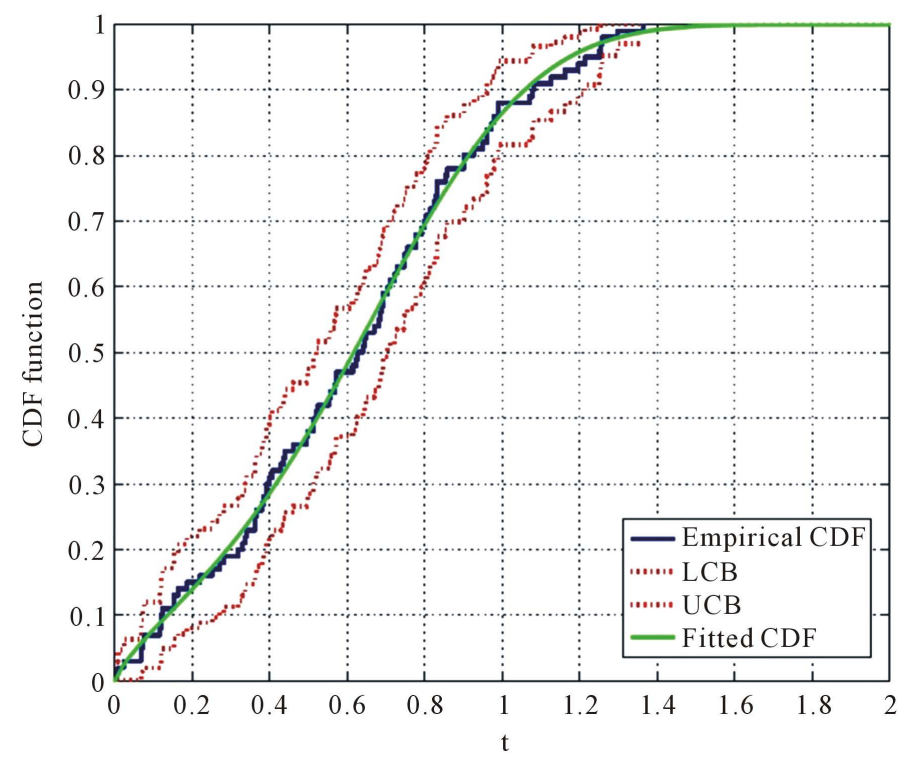

Figure 6. Cdf and empirical cdf of the $\operatorname{AddW}(\alpha, \beta, \theta, \gamma)$.

$\left(t_{1}, t_{2}, \cdots, t_{n}\right)$ be a random sample of the AddW distribution with unknown parameters $\alpha, \beta, \theta$ and $\gamma$. By setting $\Theta=(\alpha, \beta, \theta, \gamma)$, the likelihood function of this sample is given by:

$$
L\left(t_{i} ; \Theta\right)=\prod_{i=1}^{n}\left(\alpha \theta t_{i}^{\theta-1}+\beta \gamma t_{i}^{\gamma-1}\right) \exp \left(-\alpha t_{i}^{\theta}-\beta t_{i}^{\gamma}\right)
$$

The log-likelihood function has the following form:

$$
\ln L\left(t_{i} ; \Theta\right)=\sum_{i=1}^{n} \log \left(\alpha \theta t_{i}^{\theta-1}+\beta \gamma t_{i}^{\gamma-1}\right)-\left(\alpha t_{i}^{\theta}+\beta t_{i}^{\gamma}\right) .
$$

After calculating the first partial derivatives of $\ln L\left(t_{i} ; \Theta\right)$ and setting the obtained expressions equal to zero, we get the following score functions:

$$
0=\sum_{i=1}^{n} \frac{\theta t_{i}^{\theta-1}}{\alpha \theta t_{i}^{\theta-1}+\beta \gamma t_{i}^{\gamma-1}}-\sum_{i=1}^{n} t_{i}^{\theta}
$$




$$
\begin{gathered}
0=\sum_{i=1}^{n} \frac{\gamma t_{i}^{\gamma-1}}{\alpha \theta t_{i}^{\theta-1}+\beta \gamma t_{i}^{\gamma-1}}-\sum_{i=1}^{n} t_{i}^{\gamma} \\
0=\sum_{i=1}^{n} \frac{\alpha t_{i}^{\theta-1}+\alpha \theta t_{i}^{\theta-1} \log \left(t_{i}\right)}{\alpha \theta t_{i}^{\theta-1}+\beta \gamma t_{i}^{\gamma-1}}-\alpha \sum_{i=1}^{n} t_{i}^{\theta} \log \left(t_{i}\right) \\
0=\sum_{i=1}^{n} \frac{\beta t_{i}^{\gamma-1}+\beta \gamma t_{i}^{\gamma-1} \log \left(t_{i}\right)}{\alpha \theta t_{i}^{\theta-1}+\beta \gamma t_{i}^{\gamma-1}}-\beta \sum_{i=1}^{n} t_{i}^{\gamma} \log \left(t_{i}\right)
\end{gathered}
$$

To get out the MLE of the unknown parameters, we have to solve the above system of four non-linear equations with respect to $\alpha, \beta, \theta$ and $\gamma$. The solution of this system of equations is not possible in closed form, so numerical technique such as the trust region method is needed to get the MLE.

We obtain the second partial derivatives of $\ln L\left(t_{i} ; \Theta\right)$ as follows:

$$
\begin{aligned}
& \frac{\partial^{2} \ln L}{\partial \alpha^{2}}=\sum_{i=1}^{n} \frac{-\left(\theta t_{i}^{\theta-1}\right)^{2}}{\left(\alpha \theta t_{i}^{\theta-1}+\beta \gamma t_{i}^{\gamma-1}\right)^{2}} \\
& \frac{\partial^{2} \ln L}{\partial \beta^{2}}=\sum_{i=1}^{n} \frac{-\left(\gamma t_{i}^{\gamma-1}\right)^{2}}{\left(\alpha \theta t_{i}^{\theta-1}+\beta \gamma t_{i}^{\gamma-1}\right)^{2}} \\
& \frac{\partial^{2} \ln L}{\partial \theta^{2}}=\sum_{i=1}^{n} \frac{2 \alpha \beta \gamma \log \left(t_{i}\right) t_{i}^{\theta-1} t_{i}^{\gamma-1}+\alpha \beta \theta \gamma \log ^{2}\left(t_{i}\right) t_{i}^{\theta-1} t_{i}^{\gamma-1}-\left(\alpha t_{i}^{\theta-1}\right)^{2}}{\left(\alpha \theta t_{i}^{\theta-1}+\beta \gamma t_{i}^{\gamma-1}\right)^{2}}-\alpha \sum_{i=1}^{n} t_{i}^{\theta} \log ^{2}\left(t_{i}\right) \\
& \frac{\partial^{2} \ln L}{\partial \gamma^{2}}=\sum_{i=1}^{n} \frac{2 \alpha \beta \theta \log \left(t_{i}\right) t_{i}^{\theta-1} t_{i}^{\gamma-1}+\alpha \beta \theta \gamma \log ^{2}\left(t_{i}\right) t_{i}^{\theta-1} t_{i}^{\gamma-1}-\left(\beta t_{i}^{\gamma-1}\right)^{2}}{\left(\alpha \theta t_{i}^{\theta-1}+\beta \gamma t_{i}^{\gamma-1}\right)^{2}}-\beta \sum_{i=1}^{n} t_{i}^{\gamma} \log ^{2}\left(t_{i}\right) \\
& \frac{\partial^{2} \ln L}{\partial \alpha \partial \beta}=\sum_{i=1}^{n} \frac{-\theta \gamma t_{i}^{\theta-1} t_{i}^{\gamma-1}}{\left(\alpha \theta t_{i}^{\theta-1}+\beta \gamma t_{i}^{\gamma-1}\right)^{2}} \\
& \frac{\partial^{2} \ln L}{\partial \alpha \partial \theta}=\sum_{i=1}^{n} \frac{\beta \gamma t_{i}^{\theta-1} t_{i}^{\gamma-1}+\beta \theta \gamma \log \left(t_{i}\right) t_{i}^{\theta-1} t_{i}^{\gamma-1}}{\left(\alpha \theta t_{i}^{\theta-1}+\beta \gamma t_{i}^{\gamma-1}\right)^{2}}-\sum_{i=1}^{n} t_{i}^{\theta} \log \left(t_{i}\right) \\
& \frac{\partial^{2} \ln L}{\partial \alpha \partial \gamma}=\sum_{i=1}^{n} \frac{-\beta \theta t_{i}^{\theta-1} t_{i}^{\gamma-1}-\beta \theta \gamma \log \left(t_{i}\right) t_{i}^{\theta-1} t_{i}^{\gamma-1}}{\left(\alpha \theta t_{i}^{\theta-1}+\beta \gamma t_{i}^{\gamma-1}\right)^{2}} \\
& \frac{\partial^{2} \ln L}{\partial \beta \partial \theta}=\sum_{i=1}^{n} \frac{-\alpha \gamma t_{i}^{\theta-1} t_{i}^{\gamma-1}-\alpha \theta \gamma \log \left(t_{i}\right) t_{i}^{\theta-1} t_{i}^{\gamma-1}}{\left(\alpha \theta t_{i}^{\theta-1}+\beta \gamma t_{i}^{\gamma-1}\right)^{2}} \\
& \frac{\partial^{2} \ln L}{\partial \beta \partial \gamma}=\sum_{i=1}^{n} \frac{\alpha \theta t_{i}^{\theta-1} t_{i}^{\gamma-1}+\alpha \theta \gamma \log \left(t_{i}\right) t_{i}^{\theta-1} t_{i}^{\gamma-1}}{\left(\alpha \theta t_{i}^{\theta-1}+\beta \gamma t_{i}^{\gamma-1}\right)^{2}}-\sum_{i=1}^{n} t_{i}^{\gamma} \log \left(t_{i}\right) \\
& \frac{\partial^{2} \ln L}{\partial \theta \partial \gamma}=\sum_{i=1}^{n} \frac{-\left(\alpha t_{i}^{\theta-1}+\alpha \theta \log \left(t_{i}\right) t_{i}^{\theta-1}\right)\left(\beta t_{i}^{\gamma-1}-\beta \gamma \log \left(t_{i}\right) t_{i}^{\gamma-1}\right)}{\left(\alpha \theta t_{i}^{\theta-1}+\beta \gamma t_{i}^{\gamma-1}\right)^{2}}
\end{aligned}
$$

Table 2 gives the estimated parameters of 10 simulations and the mean square error of each parameter.

\section{Analysis of a Real Data Set}

In this section, we analyze a real data set to demonstrate the performance of the EW and AddW distributions in 
practice. A sample of 50 components taken from Aarset [18] has been studied. For this data set, we compare at first the results of the fits of the EW distribution (EWD) against ED, GED, RD and WD which are sub-models of the EW distribution. In the second step the fits of the AddW distribution (AddWD) will be compared against WD, MWD, and LRFD which are sub-models of the AddW distribution. Table 3 gives the often used lifetimes of 50 devices introduced by Aarset. Table 4 and Table 5 show the MLE of the parameters, the log-likelihood function values and the MSD on the one hand for the ED, RD, GED, WD, EWD and on the other hand for the WD, MWD, LRFD, and the AddW models. Table 6 and Table 7 show the observed K-S test statistic values for each models EWD and AddWD and their correspondent sub-models and the p-value for each one. Figure 7 and Figure 8 show the plots of the empirical and fitted scaled TTT-Transforms, the empirical and parametric cumulative density functions, the empirical and fitted hazard and probability density functions for the models EWD, AddWD and their correspondent submodels.

However in Figure 9 we have a comparison between the two models EW and AddW. We note that for comparison purpose, we use the mean square difference between the empirical cdf and the fitted cdf, denoted by MSD. The MSD is computed by the following relation:

$$
\operatorname{MSD}=\frac{1}{r} \sum_{i=1}^{r}\left(\hat{F}_{i}-F_{i}\right)^{2},
$$

where $\hat{F}_{i}$ and $F_{i}$ are the estimated and the empirical cdf computed at the cumulative failure times $t_{i}$ and $r$ is the size of the data set.

Based on the results shown in Table 4 and Table 5, we could deduce that:

- compared with the MSD of the ED and the WD, the EWD is not the best fit of the Aarset data;

- the MSD of the AddWD has the lowest value compared with each sub-models, so the AddWD is the best in fitting the Aarset data;

- the MSD of the AddWD is smaller than the MSD of the EWD which indicates that the AddWD fits the given data better than the EWD.

\begin{tabular}{ccccc} 
Table 2. Parameter estimates of & $\alpha=1.5, \beta=0.5, \theta=3$ and & $\gamma=0.8$. \\
\hline \multicolumn{5}{c}{ ML } \\
\hline$\hat{\alpha}$ & $\hat{\beta}$ & $\hat{\theta}$ & $\hat{\gamma}$ \\
\hline 1.5457 & 0.5406 & 3.0174 & 0.8607 \\
1.5305 & 0.5294 & 3.0610 & 0.8008 \\
1.5989 & 0.5090 & 2.9544 & 0.8543 \\
1.5923 & 0.4939 & 2.9261 & 0.8389 \\
1.4958 & 0.4909 & 3.0615 & 0.8300 \\
1.4336 & 0.5443 & 3.0996 & 0.8297 \\
1.4493 & 0.5754 & 3.0863 & 0.8433 \\
1.4821 & 0.5702 & 3.1001 & 0.8461 \\
1.4579 & 0.5419 & 2.9718 & 0.8142 \\
1.4308 & 0.4620 & 3.0760 & 0.8101 \\
\hline & 0.0034 & 0.0018 & 0.0050 & 0.0014 \\
\hline
\end{tabular}

Table 3. Lifetimes of 50 devices, Aarset.

\begin{tabular}{rrrrrrrrrrrrrrrrrrrrr}
\hline 0.1 & 0.2 & 1 & 1 & 1 & 1 & 1 & 2 & 3 & 6 & 7 & 11 & 12 & 18 & 18 & 18 & 18 & 18 \\
21 & 32 & 36 & 40 & 45 & 46 & 47 & 50 & 55 & 60 & 63 & 63 & 67 & 67 & 67 & 67 & 72 & 75 \\
79 & 82 & 82 & 83 & 84 & 84 & 84 & 85 & 85 & 85 & 85 & 85 & 86 & 86 & \\
\hline
\end{tabular}


Table 4. MLE of the parameter(s), log-likelihood function values and the MSD of sub-models of the EWD.

\begin{tabular}{cccc}
\hline The Model & Parameter estimates & $\ln L$ & MSD \\
\hline RD & $\hat{\alpha}=3.1809 \times 10^{-4}$ & -264.052 & 0.0153 \\
GED & $\hat{\alpha}=0.01870, \hat{\lambda}=0.7798$ & -239.995 & 0.0152 \\
EWD & $\hat{\alpha}=6.4800 \times 10^{-10}, \hat{\theta}=4.6900, \hat{\lambda}=0.1460$ & -229.114 & 0.0151 \\
WD & $\hat{\alpha}=0.0270, \hat{\theta}=0.9490$ & -241.002 & 0.0139 \\
ED & $\hat{\alpha}=0.0219$ & -241.089 & 0.0136 \\
\hline
\end{tabular}

Table 5. MLE of the parameter(s), log-likelihood function values and the MSD of sub-models of the AddWD.

\begin{tabular}{|c|c|c|c|}
\hline The model & Parameter estimates & $\ln L$ & MSD \\
\hline LRFD & $\hat{\alpha}=0.0140, \quad \hat{\beta}=2.4000 \times 10^{-4}$ & -238.064 & 0.0282 \\
\hline WD & $\hat{\alpha}=0.0270, \quad \hat{\theta}=0.9490$ & -241.002 & 0.0139 \\
\hline MWD & $\hat{\alpha}=0.0120, \quad \hat{\beta}=2.1590 \times 10^{-8}, \quad \hat{\gamma}=4.0140$ & -230.510 & 0.0072 \\
\hline AddWD & $\hat{\alpha}=3.9345 \times 10^{-5}, \quad \hat{\beta}=0.0860, \hat{\theta}=2.3760, \quad \hat{\gamma}=0.4114$ & -228.102 & 0.0058 \\
\hline
\end{tabular}

Table 6. The MLE of the parameter(s), K-S values and the associated p-values.

\begin{tabular}{cccc}
\hline The model & Parameter estimates & K-S & p-value \\
\hline RD & $\hat{\alpha}=3.1809 \times 10^{-4}$ & 0.2552 & 0.0328 \\
GED & $\hat{\alpha}=0.0187, \hat{\lambda}=0.7798$ & 0.1775 & 0.2678 \\
WD & $\hat{\alpha}=0.0270, \hat{\theta}=0.9490$ & 0.1657 & 0.3440 \\
ED & $\hat{\alpha}=0.0219$ & 0.1601 & 0.3846 \\
EWD & $\hat{\alpha}=6.48 \times 10^{-10}, \hat{\theta}=4.69, \hat{\lambda}=0.146$ & 0.1490 & 0.4732 \\
\hline
\end{tabular}

Table 7. The MLE of the parameter(s), K-S values and the associated p-values.

\begin{tabular}{|c|c|c|c|}
\hline The model & Parameter estimates & K-S & p-value \\
\hline LRFD & $\hat{\alpha}=0.0140, \quad \hat{\beta}=2.4000 \times 10^{-4}$ & 0.2057 & 0.1366 \\
\hline WD & $\hat{\alpha}=0.0270, \quad \hat{\theta}=0.9490$ & 0.1657 & 0.3440 \\
\hline MWD & $\hat{\alpha}=0.0120, \quad \hat{\beta}=2.159 \times 10^{-8}, \quad \hat{\lambda}=4.0140$ & 0.1655 & 0.3453 \\
\hline AddWD & $\hat{\alpha}=3.9345 \times 10^{-5}, \quad \hat{\beta}=0.0860, \quad \hat{\theta}=2.3760, \quad \hat{\lambda}=0.4114$ & 0.1230 & 0.7089 \\
\hline
\end{tabular}




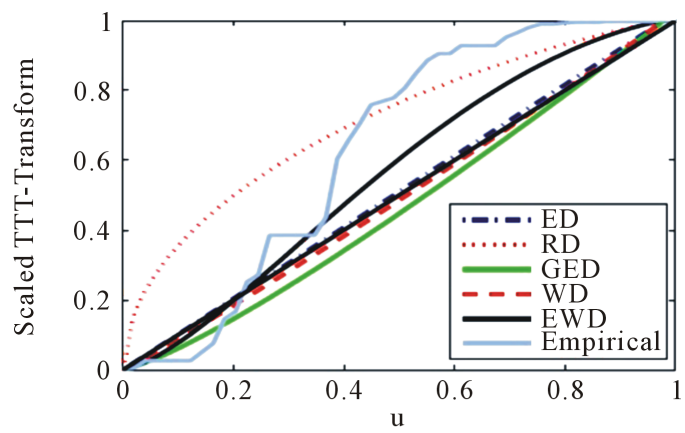

(a)

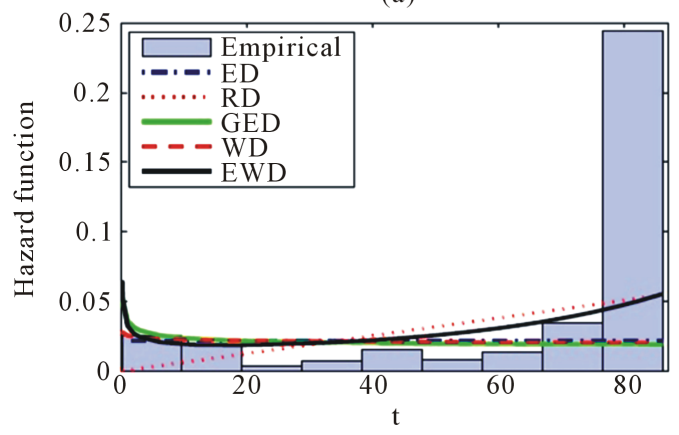

(c)

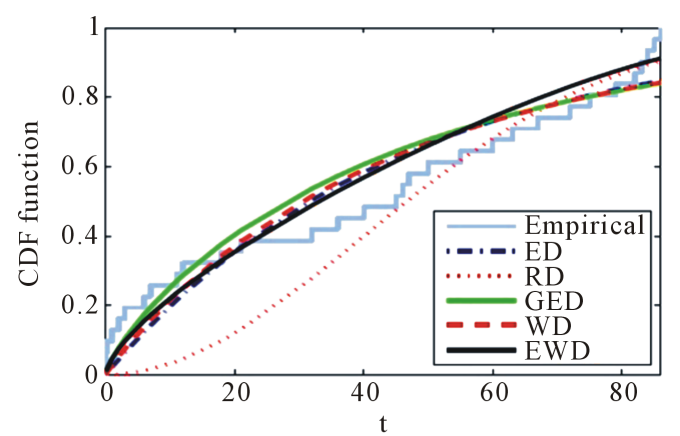

(b)

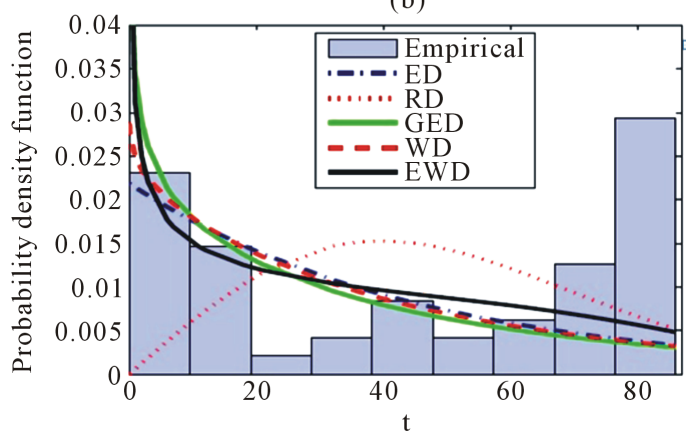

(d)

Figure 7. (a) The empirical and estimated scaled TTT-Transform plots of the ED, RD, GED, WD and EWD models; (b) The empirical and estimated cumulative density function of the ED, RD, GED, WD and EWD models; (c) Empirical and estimated hazard rate functions of the ED, RD, GED, WD and EWD models; (d) Empirical and estimated PDF of the ED, RD, GED, WD and EWD models, for Aarset data.

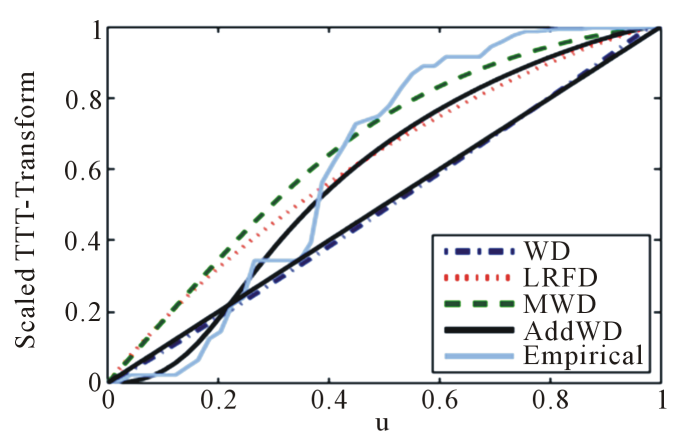

(a)

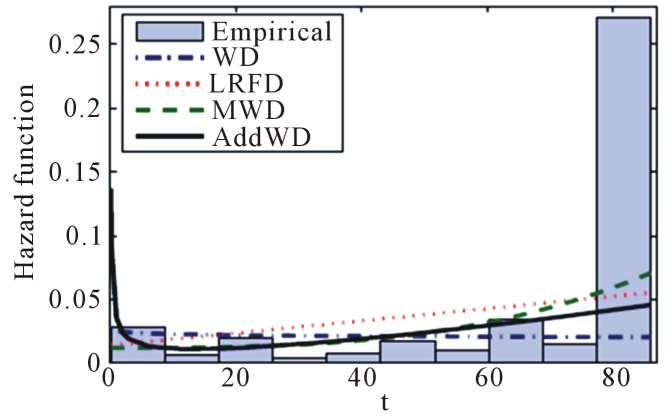

(c)

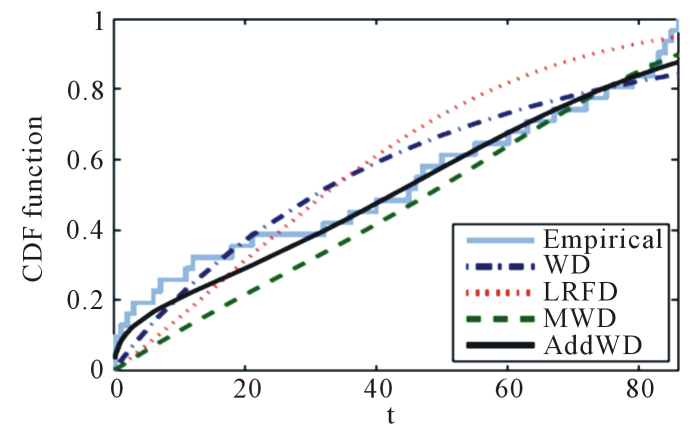

(b)

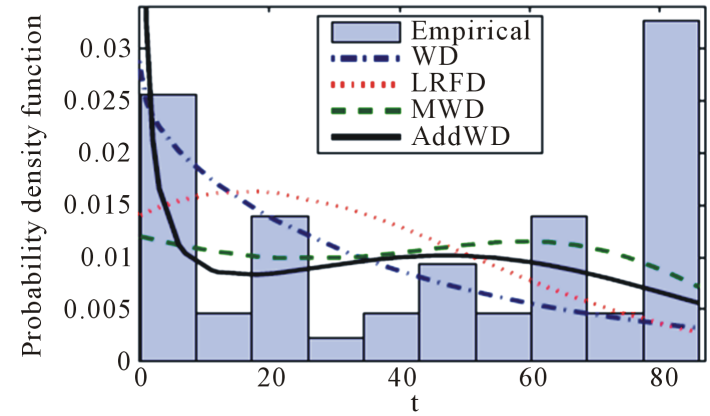

(d)

Figure 8. (a) The empirical and estimated scaled TTT-Transform plots of the WD, LRFD, MWD and AddWD models; (b) The empirical and estimated cumulative density function of the WD, LRFD, MWD and AddWD models; (c) Empirical and estimated hazard rate functions of the WD, LRFD, MWD and AddWD models; (d) Empirical and estimated pdf of the WD, LRFD, MWD and AddWD models, for Aarset data. 


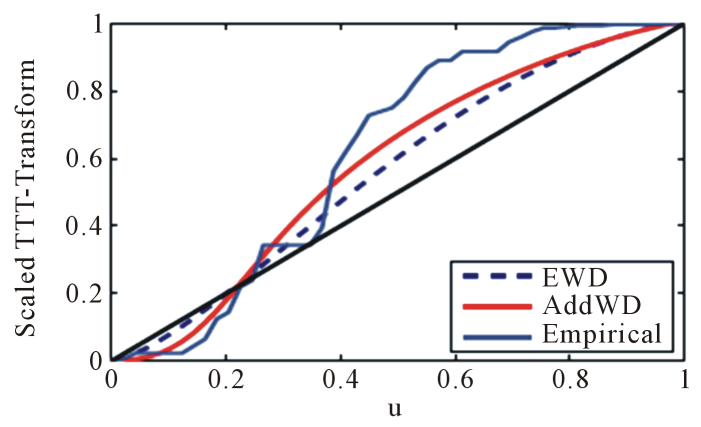

(a)

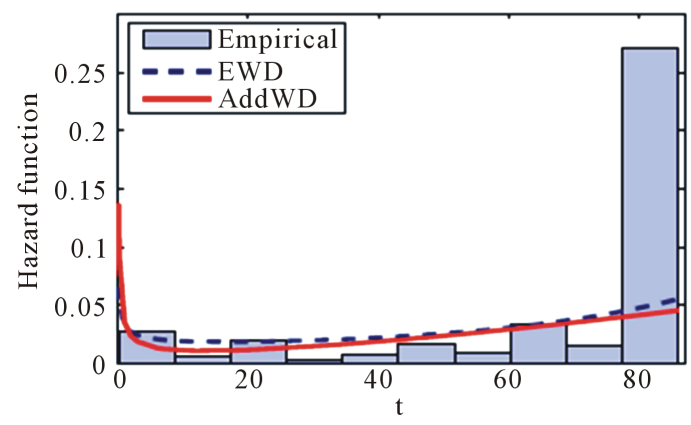

(c)

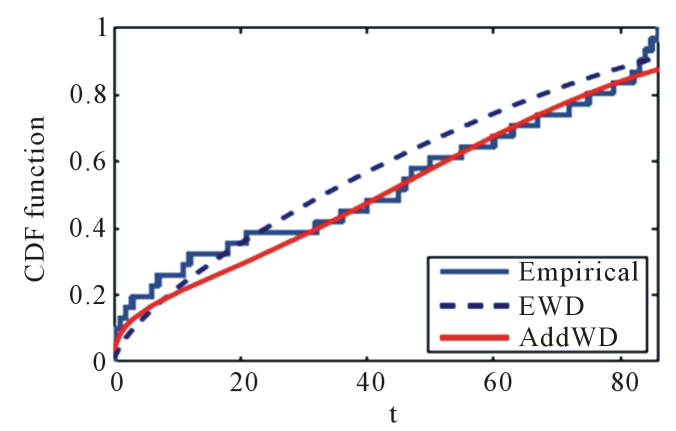

(b)

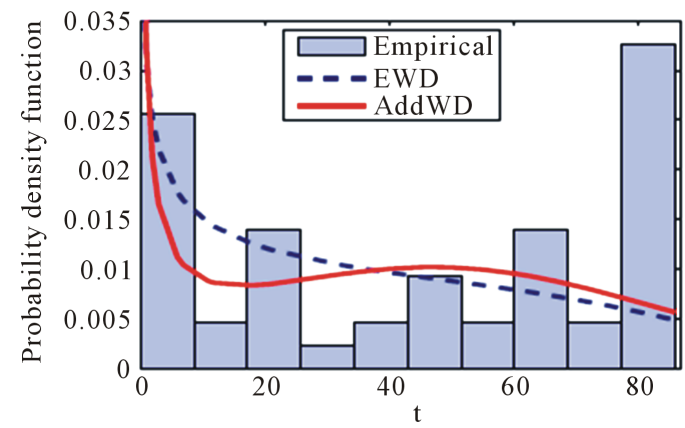

(d)

Figure 9. (a) The empirical and estimated scaled TTT-Transform plots of the EWD and AddWD models; (b) The empirical and estimated cumulative density function of the EWD and AddWD models; (c) Empirical and estimated hazard rate functions of the EWD and AddWD models; (d) Empirical and estimated PDF of the EWD and AddWD models, for Aarset data.

We perform in the next step at first the test of the following null hypotheses:

1) $H_{0}: \quad \alpha=1, \quad \lambda=1$, the data follow ED "Exponential distribution",

2) $H_{0}: \theta=2, \quad \lambda=1$, the data follow RD "Rayleigh distribution",

3) $H_{0}: \quad \theta=1$, the data follow GED "Generalized exponential distribution”,

4) $H_{0}: \quad \lambda=1$, the data follow WD "Weibull distribution",

in favor of the alternative hypothesis $H_{a}$ : the data follow the EWD "Exponentiated Weibull distribution".

And on the other hand the test of the following null hypotheses.

1) $H_{0}: \beta=0$, the data follow WD "Weibull distribution",

2) $H_{0}: \quad \theta=1, \quad \gamma>0$, the data follow MWD "Modified Weibull distribution",

3) $H_{0}: \theta=1, \quad \gamma=2$, the data follow LRFD "Linear failure rate distribution",

in favor of the alternative hypothesis $H_{a}$ : the data follow the AddWD “Additive Weibull distribution".

In the following, we use a non parametric test statistics, Kolmogorov-Smirnov (K-S) test with a level of significance equal to 0.05 , to test the null hypothesis mentioned below against $H_{a}$. We accept $H_{0}$ with the p-value under the condition p-value $>0.05$.

If we compare the EWD model with the sub-models ED, RD, GED and WD, we can conclude from Table 6 that:

- only the $\mathrm{RD}$ is rejected at level $v \geq 0.033$;

- all $H_{0}$ 's excepted the RD are not rejected at $v \leq 0.26$;

- the EWD is the best model among those discussed here, to fit the current data set because it has the biggest p-value (0.4732) and the lowest K-S value (0.1490).

Similarly, when we compare the AddWD model with the sub-models WD, LRFD and MWD, we can conclude from Table 7 that:

- none of $H_{0}$ 's is rejected at level $v \leq 0.13$;

- the AddWD is the best model among those discussed here, to fit the current data set because it has the biggest p-value (0.7089) and the lowest K-S value (0.1230);

- the AddWD is the best model among the EWD model to fit the current data set because it has the lowest K-S 
value (0.1230).

We can immediately observe from Figure 7, Figure 8 and Figure 9 that: 1) the data set has a bathtub shaped hazard rate, 2) one can see the closeness of the fitted pdf using the AddWD model, 3) the AddWD fits the data set better than all other distributions used here, because its fitted curve is closer to the empirical curve.

\section{Conclusion}

In this paper, we show the performance of two models called the exponentiated Weibull distribution and the additive Weibull distribution by using an empirical comparison with the sub-models of each one such as the exponential distribution, the Rayleigh distribution, the generalized Weibull distribution, the linear failure rate distribution, the Weibull distribution and the modified Weibull distribution. The maximum likelihood estimations of the unknown parameters for these distributions are discussed. A real data set of Aarset is studied by using the EW and the AddW distributions. The results of the comparisons showed that the additive Weibull distribution provided a better fit for the Aarset data set than some of the often-used distributions.

\section{References}

[1] Weibull, W. (1951) A Statistical Distribution Function of Wide Applicability. Journal of Applied Mechanics, 18, 293-297.

[2] Murthy, D.N.P., Xie, M. and Jiang, R. (2003) Weibull Models. John Wiley \& Sons, New York. http://dx.doi.org/10.1002/047147326X

[3] Bebbington, M., Lai, C.D. and Zitikis, R. (2007) A Flexible Weibull Extension. Reliability Engineering and System Safety, 92, 719-726. http://dx.doi.org/10.1016/j.ress.2006.03.004

[4] Zhang, T. and Xie, M. (2011) On the Upper Truncated Weibull Distribution and Its Reliability Implications. Reliability Engineering and System Safety, 96, 194-200. http://dx.doi.org/10.1016/j.ress.2010.09.004

[5] Mudholkar, G.S. and Srivastava, D.K. (1993) Exponentiated Weibull Family for Analyzing Bathtub Failure-Rate Data. IEEE Transactions on Reliability, 42, 299-302. http://dx.doi.org/10.1109/24.229504

[6] Sarhan, A.M. and Zaindin, M. (2009) Modified Weibull Distribution. Applied Sciences, 11, 123-136.

[7] Gasmi, S. and Berzig, M. (2011) Parameters Estimation of the Modified Weibull Distribution Based on Type I Censored Samples. Applied Mathematical Sciences, 59, 2899-2917.

[8] Marshall, A.W. and Olkin, I. (1997) A New Method of Adding a Parameter to a Family of Distributions with Application to the Exponential and Weibull Families. Biometrika, 84, 641-652. http://dx.doi.org/10.1093/biomet/84.3.641

[9] Xie, M., Tang, Y. and Goh, T.N. (2002) A Modified Weibull Extension with Bathtub-Shaped Failure Rate Function. Reliability Engineering and System Safety, 76, 279-285. http://dx.doi.org/10.1016/S0951-8320(02)00022-4

[10] Lai, C.D., Xie, M. and Murthy, D.N.P. (2003) Modified Weibull Model. IEEE Transactions on Reliability, 52, 33-37. http://dx.doi.org/10.1109/TR.2002.805788

[11] Xie, M. and Lai, C.D. (1996) Reliability Analysis Using an Additive Weibull Model with Bathtub Shaped Failure Rate Function. Reliability Engineering \& System Safety, 52, 87-93. http://dx.doi.org/10.1016/0951-8320(95)00149-2

[12] Famoye, F., Lee, C. and Olumolade, O. (2005) The Beta-Weibull Distribution. Journal of Statistical Theory and Applications, 4, 121-138.

[13] Cordeiro, G.M., Ortega, E.M. and Nadarajah, S. (2010) The Kumaraswamy Weibull Distribution with Application to Failure Data. Journal of the Franklin Institute, 347, 1399-1429. http://dx.doi.org/10.1016/j.jfranklin.2010.06.010

[14] Phani, K.K. (1987) A New Modified Weibull Distribution Function. Communications of the American Ceramic Society, 70, 182-184.

[15] Silva, G.O., Ortega, E.M. and Cordeiro, G.M. (2010) The Beta Modified Weibull Distribution. Lifetime Data Analysis, 16, 409-430. http://dx.doi.org/10.1007/s10985-010-9161-1

[16] Cordeiro, G.M., Nadarajah, S. and Ortega, E.M. (2013) General Results for the Beta Weibull Distribution. Journal of Statistical Computation and Simulation, 83, 1082-1114. http://dx.doi.org/10.1080/00949655.2011.649756

[17] Almalki, S.J. and Nadarajah, S. (2014) Modifications of the Weibull Distribution: A Review. Reliability Engineering and System Safety, 124, 32-55. http://dx.doi.org/10.1016/j.ress.2013.11.010

[18] Aarset, M.V. (1987) How to Identify Bathtub Hazard Rate. IEEE Transactions on Reliability, 36, $106-108$. http://dx.doi.org/10.1109/TR.1987.5222310

[19] Mudholkar, G.S., Srivastava, D.K. and Freimer, M. (1995) The Exponentiated Weibull Family: A Reanalysis of the 
Bus-Motor-Failure Data. Technometrics, 37, 436-445. http://dx.doi.org/10.1080/00401706.1995.10484376

[20] Mudholkar, G.S. and Hutson, A.D. (1996) The Exponentiated Weibull Family: Some Properties and a Flood Data Application. Communications Statistical Theory Methods, 25, 3059-3083. http://dx.doi.org/10.1080/03610929608831886

[21] Gupta, R.D. and Kundu, D. (2007) Generalized Exponential Distribution: Existing Results and Some Recent Developments. Journal of Statistical Planning and Inference, 137, 3537-3547. http://dx.doi.org/10.1016/j.jspi.2007.03.030

[22] Gasmi, S., Love, C.E. and Kahle, W. (2003) A General Repair, Proportional-Hazards, Framework to Model Complex Repairable Systems. IEEE Transactions on Reliability, 52, 26-32. http://dx.doi.org/10.1109/TR.2002.807850

[23] Gasmi, S. (2011) Parameter Estimation in an Alternating Repair Model. Journal of Statistical Planning and Inference, 141, 3605-3616. http://dx.doi.org/10.1016/j.jspi.2011.05.013 
Scientific Research Publishing (SCIRP) is one of the largest Open Access journal publishers. It is currently publishing more than 200 open access, online, peer-reviewed journals covering a wide range of academic disciplines. SCIRP serves the worldwide academic communities and contributes to the progress and application of science with its publication.

Other selected journals from SCIRP are listed as below. Submit your manuscript to us via either submit@scirp.org or Online Submission Portal.
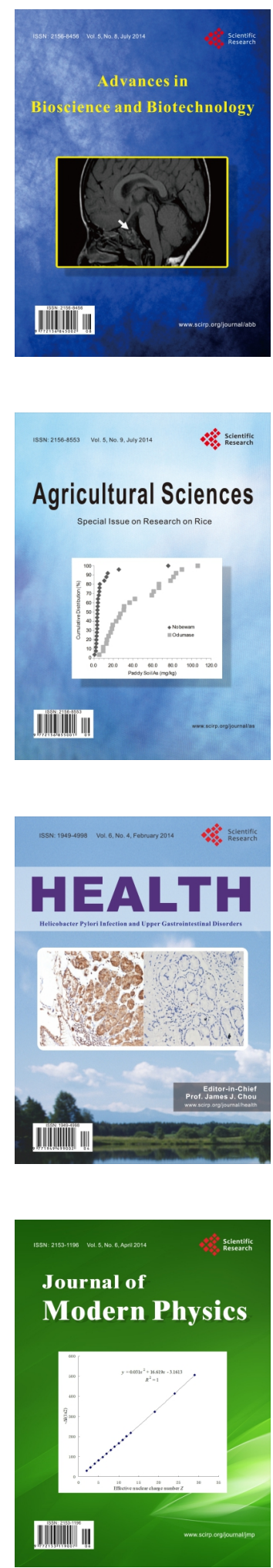
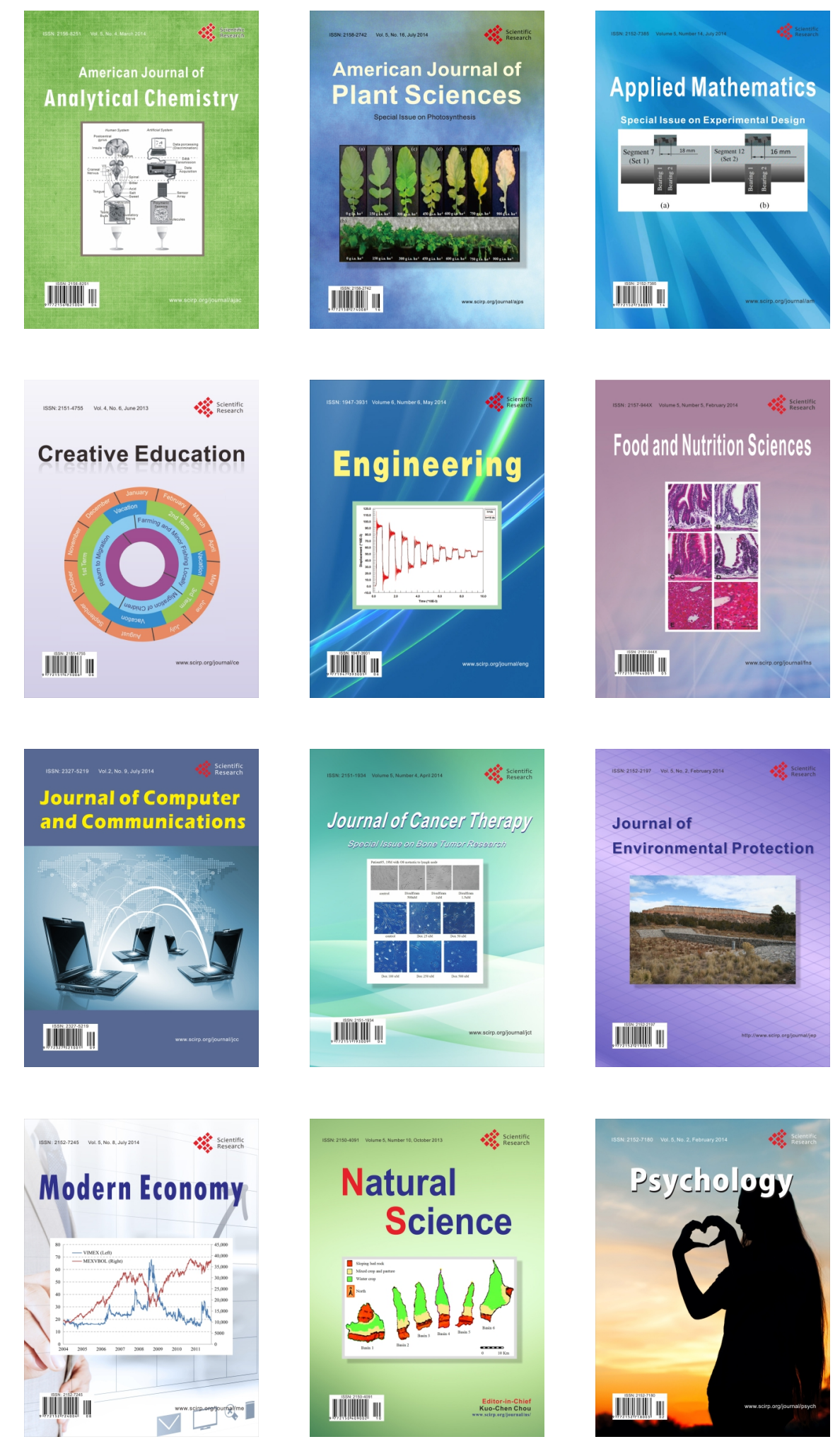